Groll

Praxis-Handbuch Erbrechtsberatung 



\title{
Praxis-Handbuch Erbrechtsberatung
}

\author{
begründet von \\ Rechtsanwalt Prof. Dr. Klaus Michael Groll
}

Ab der 4. Auflage fortgeführt und herausgegeben von

Rechtsanwalt Dr. Anton Steiner

Bearbeitet von

Prof. Dr. Stefan Edenfeld

apl. Professor an der

Westfälischen Wilhelms-Universität Münster

Dr. Thomas Endemann

Rechtsanwalt und Notar, Münster

Antje Esser

Rechtsanwältin, Neu-Ulm

Dr. Oliver Fröhler

Notar und Notariatsdirektor, Lörrach,

Lehrbeauftragter

an der Albert-Ludwigs-Universität Freiburg i. Brsg.

Paul Grötsch

Rechtsanwalt, München, Fachanwalt für Erbrecht

Anna Holzer

Rechtsanwältin, München

Prof. Dr. Peter Kindler

o. Professor an der Ludwig-Maximilians-Universität

München

Dr. Michael Kränzle

Regierungsrat, München

Lehrbeauftragter an der FHVR in Hof

Dr. Hans-Frieder Krauß

Notar, München
Dr. Dr. Robert D. v. Morgen

Rechtsanwalt, Hamburg

Fachanwalt für Erbrecht

Prof. Dr. Karlheinz Muscheler

o. Professor an der Ruhr-Universität Bochum

Prof. Dr. Susanne Nienaber, LLM.

Professorin an der Fachhochschule Bielefeld

Matthias Rösler

Rechtsanwalt, München

Fachanwalt für Erbrecht

Gerhard Ruby

Rechtsanwalt, Villingen-Schwenningen

Fachanwalt für Erbrecht und Mediator

\section{Dr. Falk Schulz}

Rechtsanwalt, Münster

Fachanwalt für Erbrecht

\section{Prof. Dr. Andreas Spickhoff}

o. Professor an der Ludwig-Maximilians-Universität München

Dr. Klaus Stein

Rechtsanwalt und Steuerberater, Osnabrück

\section{Dr. Anton Steiner}

Rechtsanwalt, München, Fachanwalt für Erbrecht

Dr. Constanze Trilsch

Rechtsanwältin, Dresden, Fachanwältin für Erbrecht

4. neubearbeitete Auflage

2015

\section{ottoschmidt}


Bibliografische Information

der Deutschen Nationalbibliothek

Die Deutsche Nationalbibliothek verzeichnet diese Publikation in der Deutschen Nationalbibliografie; detaillierte bibliografische Daten sind im Internet über http://dnb.d-nb.de abrufbar.

Verlag Dr. Otto Schmidt KG

Gustav-Heinemann-Ufer 58, 50968 Köln

Tel. 02 21/937 38-01, Fax 02 21/937 38-943

info@otto-schmidt.de

www.otto-schmidt.de

ISBN 978-3-504-18063-8

C2015 by Verlag Dr. Otto Schmidt KG, Köln

Das Werk einschließlich aller seiner Teile ist urheberrechtlich geschützt. Jede Verwertung, die nicht ausdrücklich vom Urheberrechtsgesetz zugelassen ist, bedarf der vorherigen Zustimmung des Verlages. Das gilt insbesondere für Vervielfältigungen, Bearbeitungen, Übersetzungen, Mikroverfilmungen und die Einspeicherung und Verarbeitung in elektronischen Systemen.

Das verwendete Papier ist aus chlorfrei gebleichten Rohstoffen hergestellt, holz- und säurefrei, alterungsbeständig und umweltfreundlich.

Einbandgestaltung: Jan P. Lichtenford, Mettmann

Satz: Schäper, Bonn

Druck und Verarbeitung: Kösel, Krugzell

Printed in Germany 